\title{
Numerical and experimental study of stochastic resistive switching
}

\author{
G. A. Patterson, ${ }^{1, *}$ P. I. Fierens, ${ }^{2}$ A. A. García, ${ }^{3}$ and D. F. Grosz ${ }^{2}$ \\ ${ }^{1}$ Instituto Tecnológico de Buenos Aires, Avenida E. Madero 399 (C1106ACD), C.A.B.A., Argentina \\ ${ }^{2}$ Instituto Tecnológico de Buenos Aires and Consejo Nacional de Investigaciones Científicas y Técnicas, Argentina \\ ${ }^{3}$ Facultad de Ciencias Exactas y Naturales, Universidad de Buenos Aires, Intendente Güiraldes 2160 (C1428EGA), C.A.B.A., Argentina
}

(Received 11 October 2012; published 22 January 2013)

\begin{abstract}
In this paper we study the role of noise in the context of resistive switching phenomena by means of experiments and numerical simulations. Experiments are conducted on a manganite sample. We show that the addition of external Gaussian noise to a small amplitude driving signal yields a contrast ratio between low- and high-resistance states, comparable to that obtained by the application of a large amplitude noiseless signal. Furthermore, excellent agreement between numerical simulation and measurement allows us to study resistive switching under varying input conditions and, thus, properly characterize the beneficial role of noise. We believe these results might be of relevance in the area of memory devices where the large scale of electronic integration renders the presence of noise unavoidable.
\end{abstract}

DOI: 10.1103/PhysRevE.87.012128

PACS number(s): 02.50.Ey, 05.40.Ca, 85.40.Qx

\section{INTRODUCTION}

In the past few years there has been an increased effort in the search of alternative technologies for computer memory devices. This effort is motivated by the eventual inability of current technologies to support the increase of memory storage densities as predicted by Moore's law. In this context, logic gates that work with the help of noise have been suggested (see, e.g., Refs. [1-9]). In a similar vein, memories that can benefit from the presence of noise have also been proposed (see, e.g., Refs. [10-13]).

One of the proposed alternatives for succeeding current nonvolatile memory technologies are resistive random-access memories (ReRAMs) (see, e.g., Ref. [14]). ReRAMs are based in the resistive switching phenomenon observed in several materials, that is, the change of electrical resistance by the application of electrical pulses. Often, resistive switching devices are associated with a type of two-terminal passive circuit element known as a memristor [15,16], which was originally proposed by Chua in Ref. [17]. There are different mechanisms proposed to explain the observed behavior of resistive switching materials (see, e.g., Refs. [14,15,18-22] and references therein). From a macroscopic point of view, one of the simplest models is that proposed in Ref. [15], described by

$$
\begin{gathered}
v(t)=R(x, i) i(t), \\
\frac{d x}{d t}=f(x, i),
\end{gathered}
$$

where $v(t)$ is the applied voltage, $i(t)$ is the current, $R$ is the device resistance, $x \in\left[x_{\mathrm{on}}, x_{\mathrm{off}}\right]$ is an internal state variable, and $f(x, i)$ is a nonlinear function. Among the several suggested alternatives for $f(x, i)$ (see, e.g., Ref. [23]), one commonly used is

$$
f(x, i)=4 \alpha x\left(x_{\mathrm{off}}-x\right) i,
$$

where $\alpha$ is a constant. Based on these equations, Stotland and Di Ventra [24] showed that internal noise [i.e., additive noise

\footnotetext{
*german@df.uba.ar
}

in Eq. (2)] helps increase the contrast ratio between lowand high-resistance values. This conclusion has important practical consequences, as the contrast in resistance values can be associated with the probability of error in ReRAMs.

Patterson et al. [25] extended the results of Stotland and Di Ventra [24]. In particular, they showed that external noise, that is, noise added to the externally applied voltage, does not help increase the contrast between low- and high-resistance states if Eqs. (1)-(3) are valid. However, in Sec. III we present experimental results that clearly show a positive effect of external noise. In Sec. II we describe a numerical model introduced by Pickett $e t a l$. [18] that uses a function $f(x, i)$, which depends on two threshold current values, $i_{\text {on }}$ and $i_{\text {off }}$, and leads to three different regimes in the dynamics of $x$. As it is shown in Sec. III, the model in Ref. [18] accounts well for the observed behavior.

In Sec. IV we further the analysis of the role of noise under different conditions by numerical simulations. Finally, in Sec. V, we discuss conclusions.

\section{RESISTIVE SWITCHING MODEL}

Pickett et al. [18] presented a model of the behavior of a memristor consisting of a resistor, corresponding to the bulk resistance, in series with an electron tunnel barrier formed at the interface between a metallic contact and the sample material (see Fig. 1). The width of the barrier can be modulated by applying an external field. Since a narrower barrier leads to a lower resistance, the total resistance of the device can be changed through the application of voltage pulses. While Pickett and colleagues proposed a phenomenological differential equation for the modulation of the barrier width, they used Simmons's model [26] for the calculation of the tunneling current.

The model of Pickett $e t$ al. can be described by Eqs. (1) and (2), where $x$ represents the barrier width, $f(x, i)$ is the phenomenological law that governs its evolution, and $R(x, i)$ is a complex function that takes into account the bulk resistance and the voltage dependence of the tunneling current. Kvatinsky et al. [27] presented a simplification of the model in Ref. [18], which lends itself better to numerical integration. In particular, 


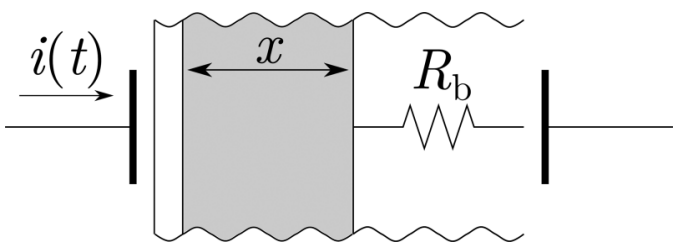

FIG. 1. The model of Pickett et al. consists of a resistor $R_{\mathrm{b}}$ in series with an electron tunnel barrier of width $x$. The modulation of $x$ via an external field leads to a change in the device resistance.

the modulation of the barrier is governed by Eq. (2) and

$$
f(x, i)=\left\{\begin{array}{lr}
k_{\mathrm{off}}\left(\frac{i}{i_{\mathrm{off}}}-1\right)^{\alpha_{\mathrm{off}}} f_{\mathrm{off}}(x) & 0<i_{\text {off }}<i \\
0 & i_{\mathrm{on}}<i<i_{\text {off }} \\
k_{\mathrm{on}}\left(\frac{i}{i_{\mathrm{on}}}-1\right)^{\alpha_{\mathrm{on}}} f_{\mathrm{on}}(x) & i<i_{\mathrm{on}}<0,
\end{array}\right.
$$

where $i_{\text {on,off }}$ are current thresholds, $k_{\text {on,off }}$ and $\alpha_{\text {on,off }}$ are parameters introduced to approximate Pickett's model. The functions $f_{\text {on,off }}(x)$ take into account the dependence on the variable $x$ forcing it to remain in the interval $\left[x_{\mathrm{on}}, x_{\mathrm{off}}\right]$, according to the equations

$$
\begin{aligned}
& f_{\text {off }}(x)=\exp \left[-\exp \left(+\frac{x-x_{\text {off }}}{w_{c}}\right)\right], \\
& f_{\text {on }}(x)=\exp \left[-\exp \left(-\frac{x-x_{\text {on }}}{w_{c}}\right)\right],
\end{aligned}
$$

where $w_{c}$ is a fitting parameter. Note that Eq. (4) depends on the sign of $i$, accounting for the fact that the experimentally observed ON and OFF switching speeds are different [28]. Kvatinsky et al. [27] further simplified the resistance in Eq. (1) to

$$
R(x, i)=R_{\mathrm{b}}+R_{\mathrm{on}} \exp \left(\lambda \frac{x-x_{\mathrm{on}}}{x_{\mathrm{off}}-x_{\mathrm{on}}}\right),
$$

where $\lambda=\ln \left(R_{\text {off }} / R_{\text {on }}\right)$, and $R_{\text {on }}$ and $R_{\text {off }}$ are the values of the sample resistance reached when the width of the barrier is $x_{\text {on }}$ and $x_{\text {off }}$, respectively.

\section{EXPERIMENTAL RESULTS}

We studied the effect of noise on a polycrystalline sample of $\mathrm{La}_{0.325} \mathrm{Pr}_{0.300} \mathrm{Ca}_{0.375} \mathrm{MnO}_{3}$ with three hand-painted silver electrodes. The behavior of this material has already been studied in, e.g., Refs. [21,22,29,30]. The experimental setup is shown in Fig. 2. Current pulses were applied through contacts A and C. From previous results $[20,31]$ it is known that the phenomenon of resistive switching takes place near both contact surfaces, $\mathrm{A}$ and $\mathrm{C}$, and that the resistance change in

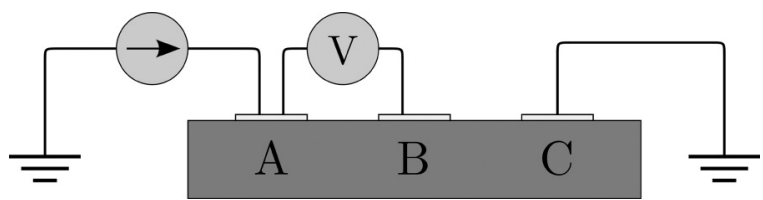

FIG. 2. Experimental setup. Current pulses were injected through contacts $\mathrm{A}$ and $\mathrm{C}$ and the device resistance was calculated by measuring the voltage drop between contacts A and B when a small bias current was applied. each surface is complementary. For this reason, we conducted four-terminal measurements, that is, we applied the current pulses between electrodes $\mathrm{A}$ and $\mathrm{C}$, changing both resistive states, and measured the voltage drop between electrodes $\mathrm{A}$ and $\mathrm{B}$, or between $\mathrm{C}$ and $\mathrm{B}$, when a small bias current was applied. Since the resistance at B does not change, the variation on the resistance measured between $\mathrm{A}$ and $\mathrm{B}$ ( $\mathrm{C}$ and $\mathrm{B}$ ) corresponds to the change of the resistance close to electrode A (C).

We applied 1-ms current pulses every $2 \mathrm{~s}$. Pulses were amplitude-modulated by a stochastic signal. The mean modulating signal was a triangular waveform with a period of $200 \mathrm{~s}$. The amplitude of this triangular waveform was itself changed in a deterministic manner: while for the first 4 cycles, the amplitude was set to $I_{\max }=600 \mathrm{~mA}$, the amplitude of the remaining 16 cycles was $I_{\max }=300 \mathrm{~mA}$. Noise was added in cycles 9 through 12 and 17 through 20. White Gaussian noise samples were computer generated at a rate of $\sim 100 \mathrm{k}$ Samples/s, standard deviation $\sim 100 \mathrm{~mA}$, with a measured effective bandwidth of $\sim 75 \mathrm{kHz}$.

Figure 3 shows the resistance measured between $\mathrm{A}$ and B for a typical realization of the experiment. As it can be readily observed in the first $1600 \mathrm{~s}$ ( 8 cycles of the triangular waveform), when the amplitude is $\leqslant 300 \mathrm{~mA}$ (cycles 4-7) the change in resistance is much smaller than that observed when the amplitude is doubled (first 4 cycles). However, resistance change can be significantly increased when external noise is added (cycles 9-12). Furthermore, the addition of noise may lead to variations of the same order of magnitude as those observed with large noiseless pulses (compare cycles 17-20 to cycles 1-4).

Figure 3 also shows the results of averaging 1000 simulations using Eqs. (1), (2), and (4)-(6). Following Ref. [27], we set the fitting parameters $k_{\mathrm{on}}=-4.68 \cdot 10^{-13} \mathrm{~nm} / \mathrm{s}, k_{\mathrm{off}}=$ $1.46 \cdot 10^{-9} \mathrm{~nm} / \mathrm{s}, \alpha_{\text {on,off }}=10, x_{\text {on }}=1.8 \mathrm{~nm}, x_{\text {off }}=1.2 \mathrm{~nm}$, and $w_{c}=107 \cdot 10^{-3} \mathrm{~nm}$. The remaining parameters were

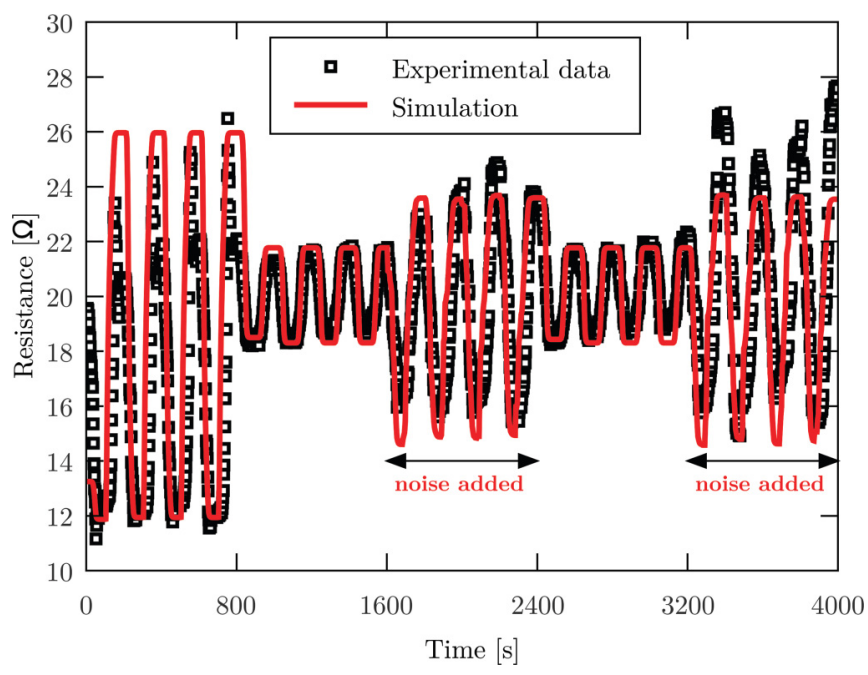

FIG. 3. (Color online) Results from a typical experimental realization and the average of 1000 simulated realizations. The addition of noise (the last $800 \mathrm{~s}$ of the time series) leads to a change in the device resistance comparable to that observed with large noiseless pulses (the first $800 \mathrm{~s}$ ). 
chosen to match the experimental data, $R_{\text {on }}=1.3 \Omega, R_{\text {off }}=$ $135 \Omega, R_{\mathrm{b}}=0 \Omega, i_{\text {on }}=-980 \mu \mathrm{A}$, and $i_{\text {off }}=11.5 \mathrm{~mA}$. These parameters render a characteristic response time of the order of $10 \mu \mathrm{s}$ (100 kHz bandwidth). Last, Gaussian noise samples were generated to resemble the experimental conditions.

Despite that the model of Pickett et al. [18] was devised for $\mathrm{TiO}_{2}$ memristive devices and that there are some differences in the behavior of titanium dioxide and manganite samples (see, e.g., Ref. [32] and references therein), Fig. 3 shows excellent agreement between experiment and simulation.

Although the noise amplitude in our experiments is of the same order of magnitude as that of the deterministic input, we can qualitatively understand the beneficial role of noise by means of a perturbation analysis. Let us consider the effect of a small current perturbation in Eq. (4),

$$
\frac{d x}{d t}=k_{\text {off }}\left(\frac{I_{0}+\varepsilon \eta_{t}}{i_{\text {off }}}-1\right)^{\alpha_{\text {off }}} f_{\text {off }}(x),
$$

where $I_{0}$ is the unperturbed constant input current, $\varepsilon\left(\ll I_{0}\right)$ is the perturbation amplitude, and $\eta_{t}$ is a zero-mean process. Without any loss of generality we assume that $I_{0} \gg i_{\text {off }}$ (a similar analysis can be carried out for the case $I_{0} \ll i_{\text {on }}$ ). Expanding Eq. (7) up to a second order in $\varepsilon$, we obtain

$$
\begin{aligned}
\frac{d x}{d t} \approx & k_{\text {off }}\left(\frac{I_{0}}{i_{\text {off }}}-1\right)^{\alpha_{\text {off }}} f_{\text {off }}(x) \\
& \times\left[1+\xi \eta_{t}+\frac{1}{2}\left(1-\alpha_{\text {off }}^{-1}\right) \xi^{2} \eta_{t}^{2}\right],
\end{aligned}
$$

where $\xi=\left(\alpha_{\text {off }} \varepsilon\right) /\left(I_{0}-i_{\text {off }}\right)$. If we assume that the correlation time of the process $\eta_{t}$ is much smaller than the characteristic time of the model, then the barrier width, $x(t)$, and $\eta_{t}$ will be nearly uncorrelated. By taking expectations on both sides of Eq. (8),

$$
\frac{d\langle x\rangle}{d t} \approx k_{\text {off }}\left(\frac{I_{0}}{i_{\text {off }}}-1\right)^{\alpha_{\text {off }}}\left\langle f_{\text {off }}(x)\right\rangle\left[1+\frac{1}{2}\left(1-\alpha_{\text {off }}^{-1}\right) \xi^{2}\right],
$$

where we used $\left\langle\eta_{t}\right\rangle=0$ and assumed that $\left\langle\eta_{t}^{2}\right\rangle=1$. Since $f_{\text {off }}(x)$ is a convex function for the allowed values of the barrier width (i.e., $x>x_{\text {off }}$ ), by Jensen's inequality we have

$$
\frac{d\langle x\rangle}{d t}>k_{\text {off }}\left(\frac{I_{0}}{i_{\text {off }}}-1\right)^{\alpha_{\text {off }}} f_{\text {off }}(\langle x\rangle)
$$

This equation states that the perturbation increases the mean velocity, leading to a larger amplitude modulation of the barrier width, which, in turn, yields a larger contrast between low- and high-resistance values.

\section{NUMERICAL SIMULATIONS}

Encouraged by the agreement between experiment and simulation shown in Fig. 3, we conducted a large number of simulations to further characterize the role of noise in resistive switching. The driving signal was now a sequence of 1-ms pulses with the pattern $I_{\max } \rightarrow 0 \rightarrow-I_{\max } \rightarrow 0$ repeated five times. Resistance was computed during the last repetition. Noise was generated as explained before and added to the driving current pulses. The pulse amplitude varied from 100 to $900 \mathrm{~mA}$ and the noise standard deviation ranged from 0 to $300 \mathrm{~mA}$.

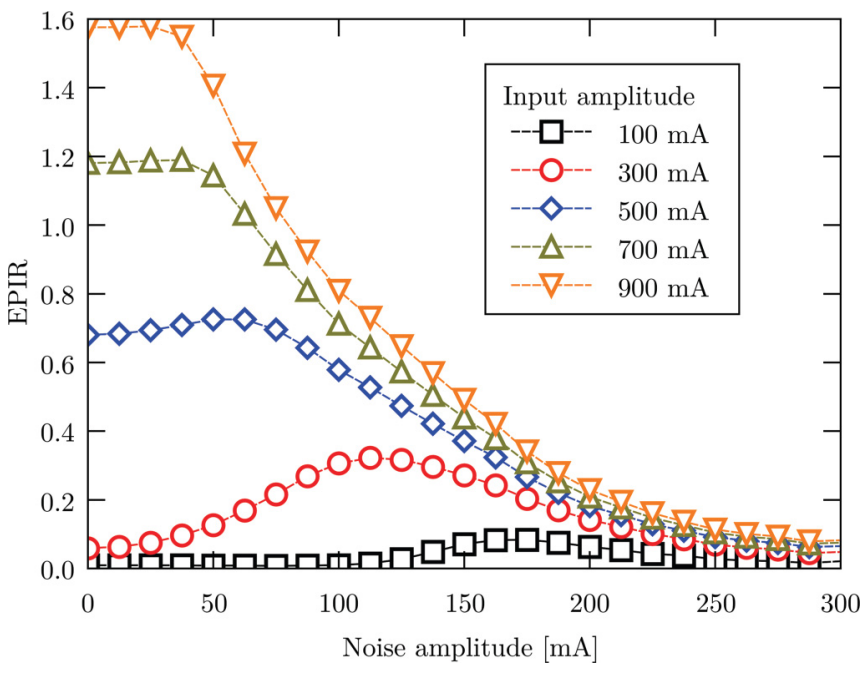

FIG. 4. (Color online) EPIR ratio as a function of noise amplitude. For small input pulses, the contrast between low- and high-resistance states is maximized for certain noise intensity. In the case of large pulses, noise only degrades the EPIR ratio.

A usual way to quantify resistance changes is by means of the electric pulse induced resistance (EPIR) ratio defined as $\left(R_{h}-R_{l}\right) / R_{l}$, where $R_{h}$ and $R_{l}$ are the high and low nonvolatile resistive states after input pulsing, respectively. Higher EPIR ratios are desired in memory applications in order to obtain a better contrast between logical states.

Figure 4 shows the EPIR ratio for varying input current and noise amplitudes. Results correspond to the average of 1000 noise realizations. As it can be observed, when large current amplitudes were applied, the addition of noise only has the effect of degrading the EPIR ratio. However, for small current amplitudes, there is a cooperative effect between the driving signal and a moderate noise intensity that leads to a higher EPIR ratio as compared to the noiseless case. In this case, increasing the noise strength will also increase the mean velocity of the tunneling barrier width, yielding a larger EPIR ratio as explained in Sec. III.

\section{CONCLUSION}

In this paper we analyzed the effect of noise on the phenomenon of resistive switching. Experiments conducted on a manganite $\left(\mathrm{La}_{0.325} \operatorname{Pr}_{0.300} \mathrm{Ca}_{0.375} \mathrm{MnO}_{3}\right)$ sample showed its beneficial role. Indeed, we showed that the addition of external noise to a low amplitude driving signal yields a contrast ratio between low- and high-resistance states, comparable to that obtained by the application of a large amplitude noiseless signal. This behavior is reminiscent of a stochastic resonant (SR) phenomenon (see, e.g., Ref. [33]) and, indeed, Stotland and Di Ventra [24] found a connection between the effect of additive Gaussian noise in resistive switching and SR.

We found excellent agreement between experimental results and numerical simulations based on a model by Pickett et al. $[18,27]$. Such agreement allowed us to properly characterize the beneficial role of noise by computing the EPIR ratio under varying input conditions. On the one hand, for small driving amplitudes, we found that the contrast between lowand high-resistance states is maximized for a certain noise 
intensity. On the other hand, for large driving signals, the presence of noise only leads to the degradation of the EPIR ratio.

Finally, we believe these results might be of significance in the area of memory devices, where the large scale of electronic integration renders the presence of noise unavoidable.

\section{ACKNOWLEDGMENTS}

The authors thank A. G. Leyva and P. Levy from CACCNEA for providing the manganite sample. We gratefully acknowledge financial support from ANPCyT under Project PICT-2010 No. 121.
[1] K. Murali, S. Sinha, W. L. Ditto, and A. R. Bulsara, Phys. Rev. Lett. 102, 104101 (2009).

[2] K. Murali, I. Rajamohamed, S. Sinha, W. L. Ditto, and A. R. Bulsara, Appl. Phys. Lett. 95, 194102 (2009).

[3] K. Murali, S. Sinha, A. Bulsara, A. Dari, and W. Ditto, in Proceedings of the International Conference on Applications in Nonlinear Dynamics (ICAND 2010), edited by V. In, A. Palacios, and P. Longhini (AIP, New York, 2011), Vol. 1339, pp. 67-77.

[4] A. R. Bulsara, A. Dari, W. L. Ditto, K. Murali, and S. Sinha, Chem. Phys. 375, 424 (2010).

[5] D. Guerra, A. Bulsara, W. Ditto, S. Sinha, K. Murali, and P. Mohanty, Nano Lett. 10, 1168 (2010).

[6] K. P. Singh and S. Sinha, Phys. Rev. E 83, 046219 (2011).

[7] A. Dari, A. Bulsara, W. Ditto, and X. Wang, in Biomedical Circuits and Systems Conference (BioCAS 2011) (IEEE, New York, 2011), pp. 337-340.

[8] A. Dari, B. Kia, X. Wang, A. R. Bulsara, and W. Ditto, Phys. Rev. E 83, 041909 (2011).

[9] A. Dari, B. Kia, A. R. Bulsara, and W. Ditto, Europhys. Lett. 93, 18001 (2011).

[10] S. A. Ibáñez, P. I. Fierens, R. P. J. Perazzo, G. A. Patterson, and D. F. Grosz, Eur. Phys. J. B 76, 49 (2010).

[11] P. I. Fierens, S. Ibáñez, R. P. J. Perazzo, G. A. Patterson, and D. F. Grosz, Phys. Lett. A 374, 2207 (2010).

[12] P. I. Fierens, G. Bellomo, G. A. Patterson, and D. F. Grosz, in Proceedings of the International Conference on Applications in Nonlinear Dynamics (ICAND 2010), edited by V. In, A. Palacios, and P. Longhini (AIP, New York, 2011), Vol. 1339.

[13] G. Bellomo, G. A. Patterson, P. I. Fierens, and D. F. Grosz, Phys. Lett. A 375, 3233 (2011).

[14] A. Sawa, Materials Today 11, 28 (2008).

[15] D. B. Strukov, G. S. Snider, D. R. Stewart, and R. S. Williams, Nature (London) 453, 80 (2008).
[16] L. Chua, Proc. IEEE 100, 1920 (2012).

[17] L. Chua, IEEE Trans. Circuit Theory 18, 507 (1971).

[18] M. D. Pickett, D. B. Strukov, J. L. Borghetti, J. J. Yang, G. S. Snider, D. R. Stewart, and R. S. Williams, J. Appl. Phys. 106, 074508 (2009).

[19] M. J. Sánchez, M. J. Rozenberg, and I. H. Inoue, Appl. Phys. Lett. 91, 252101 (2007).

[20] M. J. Rozenberg, M. J. Sánchez, R. Weht, C. Acha, F. GomezMarlasca, and P. Levy, Phys. Rev. B 81, 115101 (2010).

[21] N. Ghenzi, M. Sánchez, F. Gomez-Marlasca, P. Levy, and M. Rozenberg, J. Appl. Phys. 107, 093719 (2010).

[22] F. Gomez-Marlasca, N. Ghenzi, P. Stoliar, M. J. Sanchez, M. J. Rozenberg, G. Leyva, and P. Levy, Appl. Phys. Lett. 98, 123502 (2011).

[23] Y. N. Joglekar and S. J. Wolf, Eur. J. Phys. 30, 661 (2009).

[24] A. Stotland and M. Di Ventra, Phys. Rev. E 85, 011116 (2012).

[25] G. A. Patterson, P. I. Fierens, and D. F. Grosz, Proceedings of the Third International Conference on Applications in Nonlinear Dynamics (ICAND 2012) (in press).

[26] J. G. Simmons, J. Appl. Phys. 34, 1793 (1963).

[27] S. Kvatinsky, E. G. Friedman, A. Kolodny, and U. C. Weiser, IEEE Trans. Circuit Syst. 60, 211 (2013).

[28] D. B. Strukov, J. L. Borghetti, and R. S. Williams, Small 5, 1058 (2009).

[29] F. Gomez-Marlasca and P. Levy, J. Phys. Conf. Ser. 167, 012036 (2009).

[30] N. Ghenzi, M. Sánchez, M. Rozenberg, P. Stoliar, F. Marlasca, D. Rubi, and P. Levy, J. Appl. Phys. 111, 084512 (2012).

[31] C. Acha, Physica B 404, 2746 (2009).

[32] F. Gomez-Marlasca, N. Ghenzi, M. J. Rozenberg, and P. Levy, Appl. Phys. Lett. 98, 042901 (2011).

[33] L. Gammaitoni, P. Hänggi, P. Jung, and F. Marchesoni, Rev. Mod. Phys. 70, 223 (1998). 